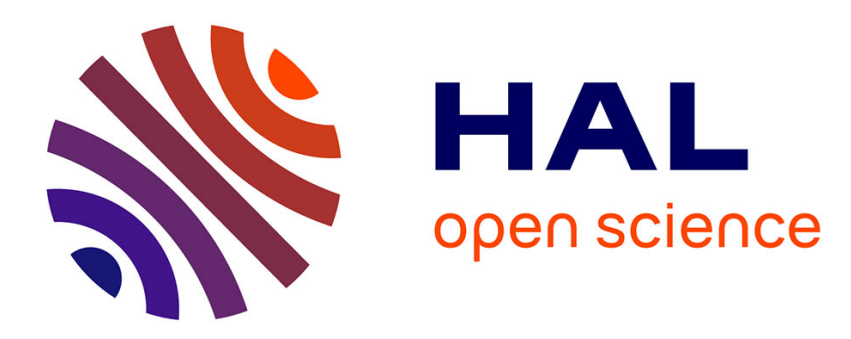

\title{
The influence of initial stress on elastic waves
}

Maurice A. Biot

\section{To cite this version:}

Maurice A. Biot. The influence of initial stress on elastic waves. Journal of Applied Physics, 1940, 11 (8), pp. 522-530. 10.1063/1.1712807 . hal-01368866

\section{HAL Id: hal-01368866 https://hal.science/hal-01368866}

Submitted on 21 Sep 2016

HAL is a multi-disciplinary open access archive for the deposit and dissemination of scientific research documents, whether they are published or not. The documents may come from teaching and research institutions in France or abroad, or from public or private research centers.
L'archive ouverte pluridisciplinaire HAL, est destinée au dépôt et à la diffusion de documents scientifiques de niveau recherche, publiés ou non, émanant des établissements d'enseignement et de recherche français ou étrangers, des laboratoires publics ou privés. 


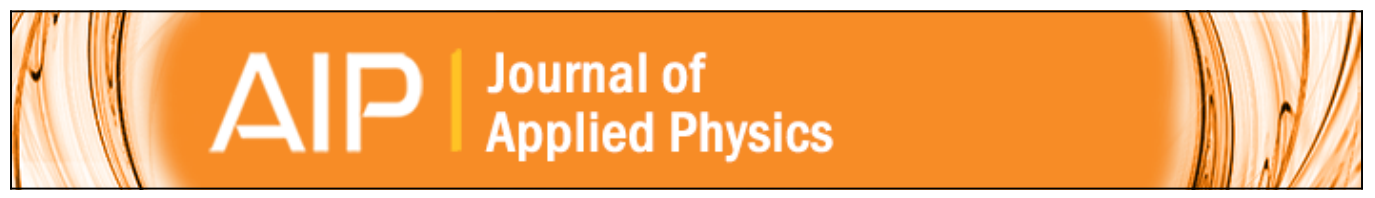

\section{The Influence of Initial Stress on Elastic Waves}

Maurice A. Biot

Citation: Journal of Applied Physics 11, 522 (1940); doi: 10.1063/1.1712807

View online: http://dx.doi.org/10.1063/1.1712807

View Table of Contents: http://scitation.aip.org/content/aip/journal/jap/11/8?ver=pdfcov

Published by the AIP Publishing

\section{Articles you may be interested in}

Propagation of Love waves in an initially stressed medium consisting of a slow elastic layer lying over a liquid-saturated porous solid half-space

J. Acoust. Soc. Am. 89, 2584 (1991); 10.1121/1.400697

Reflection of elastic waves under initial stress at a free surface: $\mathrm{P}$ and $\mathrm{S} \mathrm{V}$ motion

J. Acoust. Soc. Am. 72, 255 (1982); 10.1121/1.387987

Effect of Initial Stress on the Propagation of Flexural Waves in Elastic Rectangular Bars

J. Acoust. Soc. Am. 52, 178 (1972); 10.1121/1.1982116

Stress Waves in Nonhomogeneous Elastic Rods

J. Acoust. Soc. Am. 45, 1273 (1969); 10.1121/1.1911599

Acoustic-Gravity Waves as a Particular Case of the Theory of Elasticity under Initial Stress

Phys. Fluids 6, 778 (1963); 10.1063/1.1706813

\section{High Energy Nanosecond Lasers}
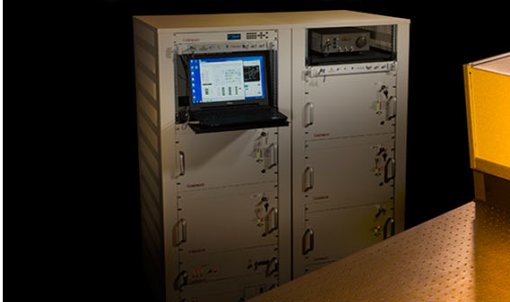


\title{
The Influence of Initial Stress on Elastic Waves*
}

\author{
MAURICE A. Biot \\ Columbia University, New York, New York
}

(Received March 27, 1940)

\begin{abstract}
A rigorous treatment is given of the problem of wave propagation in an elastic continuum when the influence of the initial stress is taken into account. After a short review of the theory various cases of initial stress are considered. It is shown that a uniform hydrostatic pressure does not change the laws of propagation. A hydrostatic pressure gradient produces a buoyancy effect which causes coupling between rotational and dilatational waves. Bromwich's equations for the effect of gravity on Rayleigh waves are derived from the general theory and the physical transition from Rayleigh waves in a very rigid medium to pure gravity waves in a liquid is discussed. The case of the vertical uniform stress is also considered and it is shown that the effect of the initial stress on the waves in this case cannot be accounted for by elastic anisotropy alone. Reflections may be produced by a discontinuity in stress without discontinuity of elastic properties.
\end{abstract}

\section{INTRODUCTION}

A SIMPLE experiment will show that initial stress must have an influence on elastic wave propagation. Consider a uniform rod held between two hinges. The oscillations of this rod obey the well-known equation

$$
E I d^{4} w / d x^{4}-\rho \omega^{2} w=0,
$$

where $w$ is the deflection, $E I$ the stiffness, $\rho$ the mass per unit length, $\omega / 2 \pi$ the frequency and $x$ is the abscissa along the rod. The fundamental mode for the hinged rod of length $l$ is

$$
w=A \sin \frac{\pi x}{l}
$$

and the corresponding frequency

$$
\frac{\omega}{2 \pi}=\frac{\pi}{2 l^{2}}\left(\frac{E I}{\rho}\right)^{\frac{1}{2}} .
$$

If there is an axial compression $P$ in the rod the equation for the free oscillations becomes

$$
E I \frac{d^{4} w}{d x^{4}}+P \frac{d^{2} w}{d x^{2}}-\rho \omega^{2} w=0 .
$$

The shape of the fundamental mode is the same as before but the frequency is now

$$
\frac{\omega}{2 \pi}=\frac{\pi}{2 l^{2}}\left(\frac{E I}{\rho}\left(1-P / P_{c}\right)\right)^{\frac{1}{2}}
$$

* Publication assisted by the Ernest Kempton Adams Fund for Physical Research of Columbia University. with

$$
P_{c}=E I \pi^{2} / l^{2} .
$$

The axial compression $P$ decreases the frequency. When $P$ approaches $P_{c}$, which is the buckling load of the rod, the frequency falls to zero.

If $P$ is negative we have an axial tension in the rod. In that case the frequency is increased. Considering a very slender rod with vanishing stiffness $(E I \rightarrow 0)$ the frequency becomes

$$
\frac{\omega}{2 \pi}=\frac{1}{2 l}\left(\frac{-P}{\rho}\right)^{\frac{1}{2}},
$$

which is the frequency for a string under a tension $-P$.

When we put the rod under tension it acquires partly the properties of a stretched string. From the viewpoint of wave propagation a tension in the rod increases the velocity of propagation and a compression decreases this velocity.

It is clear that these phenomena must be a particular aspect of the more general case of elastic wave propagation in three dimensions in a body under initial stress.

The effect of initial stress on oscillations and wave propagation has been investigated by a number of writers, but because of the lack of a general and rigorous theory the results are restricted to the case of an initial hydrostatic pressure and are of ten contradictory or incomplete. L. Brillouin's ${ }^{1}$ treatment leads to the conclusion that at sufficiently high pressure the wave

\footnotetext{
I L. Brillouin, Ann. de physique 4, 528 (1925).
} 
velocities would reduce to zero. This paradoxical result is due to incorrect assumptions. In connection with problems in geophysics the effect of initial stress on the oscillations of a gravitating sphere has been considered. Correct equations have been derived by Love $^{2}$ for the case of an incompressible material under hydrostatic pressure. He also points out that the compressibility must introduce an important effect of buoyancy but does not attempt to establish any theory for this case. By an extension of Love's method Macelwane and Sohon $^{3}$ have established equations for the oscillations of a compressible gravitating sphere. These equations may be shown to be particular cases of our general theory if we introduce explicitly the change of body force due to the oscillations of the earth itself. The effect of gravity on Rayleigh waves and the oscillations of an elastic globe was investigated by T. J. I'A Bromwich, ${ }^{4}$ applying the equations derived by Love for an incompressible material under hydrostatic pressure. In a recent paper $\mathrm{F}$. $\mathrm{Birch}^{5}$ has applied Murnaghan's ${ }^{6}$ theory of finite strain and shows that the hydrostatic pressure has no effect on the laws of propagation. This paper is restricted not only by the fact that the initial stress is assumed hydrostatic, but also by a condition not mentioned explicitly by the writer, namely that there is no initial pressure gradient. Further restriction is due to Murnaghan's assumption that the material in the unstressed state is homogeneous and isotropic, with a definite potential energy function of the finite strain so that the initial hydrostatic pressure condition is assumed to be obtained from an initial unstressed condition through a reversible process. There is no physical basis for this assumption because it is very likely that the state of hydrostatic pressure inside the earth is produced by a slow process of

${ }^{2}$ A. E. H. Love, The Mathematical Theory of Elasticity (Cambridge Univ. Press, 4th edition, 1927), pp. 176-178.

${ }^{3} \mathrm{~J}$. B. Macelwane and F. W. Sohon, Introduction to Theoretical Seismology, Part I Geodynamics (John Wiley \& Sons, New York, 1936).

${ }^{4}$ T. J. I'A Bromwich, "On the influence of gravity on elastic waves and in particular on the vibrations of an elastic globe," Proc. London Math. Soc. 30, 98-120 (1898).

${ }^{\circ} \mathrm{F}$. Birch, "The effect of pressure upon the elastic parameters of isotropic solid according to Murnaghan's theory of finite strain," J. App. Phys., 9279 (1938).

${ }^{6} \mathrm{~F}$. D. Murnaghan, "Finite deformations of an elastic solid," Am. J. Math. April, 1937. creep in which viscosity and physical-chemical irreversible processes are predominant.

The present author has derived ${ }^{7-9}$ a general theory of elasticity for bodies under initial stress which can be immediately applied to the problem of wave propagation.

No assumption is made on how the initial state of stress is produced. These initial stress components satisfy only the conditions of internal equilibrium. We give a brief review of the theory in the case of two-dimensional strain. The derivation is quite elementary and does not involve the use of tensor calculus. The equations are applied to various cases of initial stress. We find that a homogeneous hydrostatic pressure does not affect the laws of wave propagation. When there is an initial pressure gradient we show that there is coupling between rotational and compression waves. This coupling is due to a buoyancy effect whose presence was suspected by Love $^{2}$ and which we derive here in a quantitatively correct form. This effect is of course small for usual earthquake waves, but becomes preponderant if we consider tidal waves or the modes of oscillation of the earth. In the next paragraph we consider the special case of surface waves or Rayleigh waves, assuming an incompressible material with hydrostatic pressure due to gravity.

Our theory in this case leads exactly to Bromwich's equations, but a special feature of the surface waves is pointed out which is not mentioned in Bromwich's paper. Finally, we consider an initial state of stress which is homogeneous but not hydrostatic, i.e., we assume unequal initial principal stresses. This approximates the state of stress near the surface of the earth. It is found in this case that the behavior of the transversal wave alone is affected by the stress. The behavior of these waves cannot be accounted for by elastic anisotropy or a change in elastic coefficients and the existence of stress introduces an essentially new aspect in the nature

7 M. A. Biot, "Theory of elasticity with large displacements and rotations," Proc. Fifth Internat. Congress of Applied Mechanics, 1938.

${ }^{8}$ M. A. Biot, "Non linear theory of elasticity and the linearized case for a body under initial stress," Phil. Mag. [7] 27, 468 (1939).

${ }^{9}$ M. A. Biot, "Théorie de l'Elasticité du second ordre avec application à la théorie du flambage," Ann. de la Société Scientifique de Bruxelles, Serie I 59, 104 (1939). 
of the wave propagation. The velocity of propagation depends on the stress and it is possible to obtain reflections in a medium which has uniform elastic properties but contains discontinuities of initial stress. These facts must have their importance for the interpretation of data in methods of seismic prospecting.

\section{General Theory}

Consider a state of initial stress such that a principal direction is always parallel with the $z$ axis, while the small additional strains are assumed to constitute a state of plane strain in the $x y$ plane.

The initial stress is defined by the components $S_{11}, S_{22}$ and $S_{12}$ referred to rectangular axes $x, y$. They satisfy the equilibrium conditions

$$
\begin{aligned}
& \frac{\partial S_{11}}{\partial x}+\frac{\partial S_{12}}{\partial y}+\rho(x, y) X(x, y)=0, \\
& \frac{\partial S_{12}}{\partial x}+\frac{\partial S_{22}}{\partial y}+\rho(x, y) Y(x, y)=0,
\end{aligned}
$$

where $\rho$ is the specific mass and $X, Y$ the components of the body force per unit mass. An elastic element of coordinates $x, y$ acquires the coordinates $\xi=x+u, \eta=y+v$ after deformation and rotates through an angle

$$
\omega=\frac{1}{2}(\partial v / \partial x-\partial u / \partial y) .
$$

The stress components after deformation referred to directions which rotate with the material are

$$
\begin{aligned}
& \sigma_{11}=S_{11}+s_{11}, \\
& \sigma_{22}=S_{22}+s_{22}, \\
& \sigma_{12}=S_{12}+s_{12} .
\end{aligned}
$$

The components $s_{11}, s_{22}$ and $s_{12}$ of the stress increment depend only on the strain. This stress may be referred to the original $x, y$ directions and the components then become in first approximation

$$
\begin{aligned}
& \sigma_{x x}=s_{11}-2 S_{11} \omega, \\
& \sigma_{y y}=s_{22}+2 S_{22} \omega, \\
& \sigma_{x y}=s_{12}+\left(S_{11}-S_{22}\right) \omega .
\end{aligned}
$$

These are the stresses at the point $\xi, \eta$ along the $x$ and $y$ directions.

These components satisfy the dynamical equilibrium relations

$$
\begin{aligned}
& \partial \sigma_{x x} / \partial \xi+\partial \sigma_{x y} / \partial \eta+\mu(\xi, \eta) X(\xi, \eta)=\mu(\xi, \eta) \partial^{2} u / \partial t^{2} \\
& \partial \sigma_{x y} / \partial \xi+\partial \sigma_{y y} / \partial \eta+\mu(\xi, \eta) Y(\xi, \eta)=\mu(\xi, \eta) \partial^{2} v / \partial t^{2}
\end{aligned}
$$

where $\mu(\xi, \eta)$ is the specific mass after deformation.

Now we can express these equations in terms of the independent variable $x, y$ by using transformations of the type

$$
\frac{\partial \sigma_{x x}}{\partial \xi}=\frac{\partial \sigma_{x x}}{\partial x} \frac{\partial x}{\partial \xi}+\frac{\partial \sigma_{x x}}{\partial y} \frac{\partial y}{\partial \xi}, \quad \text { etc. }
$$

The partial derivatives of $x, y$ with respect to $\xi, \eta$ are in first approximation

where $D$ is the Jacobian

$$
\begin{array}{ll}
\frac{\partial x}{\partial \xi}=\frac{1}{D}\left(1+\frac{\partial v}{\partial y}\right), & \frac{\partial x}{\partial \eta}=-\frac{1}{D} \frac{\partial u}{\partial y} \\
\frac{\partial y}{\partial \xi}=-\frac{1}{D} \frac{\partial v}{\partial x}, & \frac{\partial y}{\partial \eta}=\frac{1}{D}\left(1+\frac{\partial u}{\partial x}\right)
\end{array}
$$

$$
D=\frac{d(\xi, \eta)}{d(x, y)}=\left|\begin{array}{cc}
1+\frac{\partial u}{\partial x} & \frac{\partial u}{\partial y} \\
\frac{\partial v}{\partial x} & 1+\frac{\partial v}{\partial y}
\end{array}\right|
$$


Using these relations and the property

$$
\rho(x, y)=D \mu(\xi, \eta)
$$

expressing the conservation of mass Eqs. (10) become

$$
\begin{aligned}
& \frac{\partial \sigma_{x x}}{\partial x}+\frac{\partial \sigma_{x y}}{\partial y}+e_{y y} \frac{\partial \sigma_{x x}}{\partial x}+e_{x x} \frac{\partial \sigma_{x y}}{\partial y}-\left(e_{x y}-\omega\right) \frac{\partial \sigma_{x y}}{\partial x}-\left(e_{x y}+\omega\right) \frac{\partial \sigma_{x x}}{\partial y}+\rho X(\xi, \eta)=\rho \frac{\partial^{2} u}{\partial t^{2}} \\
& \frac{\partial \sigma_{x y}}{\partial x}+\frac{\partial \sigma_{y y}}{\partial y}+e_{y y} \frac{\partial \sigma_{x y}}{\partial x}+e_{x x} \frac{\partial \sigma_{y y}}{\partial y}-\left(e_{x y}-\omega\right) \frac{\partial \sigma_{y y}}{\partial x}-\left(e_{x y}+\omega\right) \frac{\partial \sigma_{x y}}{\partial y}+\rho Y(\xi, \eta)=\rho \frac{\partial^{2} v}{\partial t^{2}}
\end{aligned}
$$

in which $e_{x x}=\partial u / \partial x, e_{y y}=\partial v / \partial y, e_{x y}=\frac{1}{2}(\partial v / \partial x+\partial u / \partial y)$ are the strain components. We now substitute the values (9) for $\sigma_{x x}, \sigma_{y y}, \sigma_{x y}$ and drop terms of higher order than the first; the above equations become

$\frac{\partial s_{11}}{\partial x}+\frac{\partial s_{12}}{\partial y}+\rho u \frac{\partial X}{\partial x}+\rho v \frac{\partial X}{\partial y}+\rho \omega Y-2 S_{12} \frac{\partial \omega}{\partial x}+\left(S_{11}-S_{22}\right) \frac{\partial \omega}{\partial y}$

$$
+e_{y y} \frac{\partial S_{11}}{\partial x}+e_{x x} \frac{\partial S_{12}}{\partial y}-e_{x y}\left(\frac{\partial S_{11}}{\partial y}+\frac{\partial S_{12}}{\partial x}\right)=\rho \frac{\partial^{2} u}{\partial t^{2}}
$$

$\frac{\partial s_{12}}{\partial x}+\frac{\partial s_{22}}{\partial y}+\rho u \frac{\partial Y}{\partial x}+\rho v \frac{\partial Y}{\partial y}+\rho \omega X+\left(S_{11}-S_{22}\right) \frac{\partial \omega}{\partial x}+2 S_{12} \frac{\partial \omega}{\partial y}$

$$
+e_{y y} \frac{\partial S_{12}}{\partial x}+e_{x x} \frac{\partial S_{22}}{\partial y}-e_{x y}\left(\frac{\partial S_{12}}{\partial y}+\frac{\partial S_{22}}{\partial x}\right)=\rho \frac{\partial^{2} v}{\partial t^{2}} .
$$

In order to obtain these equations account must be taken of the initial equilibrium conditions (6) and the identities

$$
\begin{aligned}
& \partial e_{x x} / \partial y=(\partial / \partial x)\left(e_{x y}-\omega\right), \\
& \partial e_{y y} / \partial x=(\partial / \partial y)\left(e_{x y}+\omega\right) .
\end{aligned}
$$

We remember that the stress increments $s_{11}, s_{22}, s_{12}$, depend only on the strain. They may be taken as linear functions of the strain components

$$
\begin{aligned}
& s_{11}=B_{11} e_{x x}+B_{12} e_{y y}+B_{13} e_{x y}, \\
& s_{22}=B_{21} e_{x x}+B_{22} e_{y y}+B_{23} e_{x y}, \\
& s_{12}=B_{31} e_{x x}+B_{32} e_{y y}+B_{33} e_{x y} .
\end{aligned}
$$

Assuming the existence of a potential energy function of the strain it is possible to prove ${ }^{8}$ that the elastic coefficients must satisfy the relations

$$
\begin{aligned}
& B_{12}+S_{11}=B_{21}+S_{22}, \\
& B_{13}-S_{12}=B_{31}+\frac{1}{2} S_{12}, \\
& B_{23}-S_{12}=B_{32}+\frac{1}{2} S_{12} .
\end{aligned}
$$

It is only in case of initial hydrostatic pressure $\left(S_{11}=S_{22}, S_{12}=0\right)$ that the elastic coefficients will be symmetric $\left(B_{i j}=B_{j i}\right)$. 
The boundary conditions along an element $d x, d y$ of the boundary contour are found to be

$$
\begin{aligned}
& \left(s_{11}-S_{12} \omega\right) d y-\left(s_{12}-S_{22} \omega\right) d x=d F_{x}, \\
& \left(s_{12}+S_{11} \omega\right) d y-\left(s_{22}+S_{12} \omega\right) d x=d F_{y},
\end{aligned}
$$

where $d F_{x}, d F_{y}$ are the projections of the force acting on the boundary element $d x, d y$.

For a three-dimensional theory the following equations may be derived as above. We find

$$
\sum^{\nu} \frac{\partial s^{\nu i}}{\partial x^{\nu}}-\rho \sum^{\mu} \omega_{\mu}^{i} X^{\mu}+\rho \sum^{\mu} u^{\mu} \frac{\partial X^{i}}{\partial x^{\mu}}+\rho \Delta X^{i}+\sum^{\mu \nu}\left(S^{\nu i} \frac{\partial \omega_{\nu}{ }^{\mu}}{\partial x^{\mu}}+S^{\mu \nu} \frac{\partial \omega_{\mu}{ }^{i}}{\partial x^{\nu}}\right)-e \rho X^{i}-\sum^{\mu \nu} e_{\mu}^{\nu} \frac{\partial S^{\mu i}}{\partial x^{\nu}}=\rho \frac{\partial^{2} u^{\mu}}{\partial i^{2}} .
$$

With the following notations: $x^{\nu}=$ the Cartesian coordinates, $u^{\nu}=$ the displacements, $X^{\nu}=$ the components of the initial body force, $\Delta X^{\nu}$ the increment of body force due to the motion, $\omega_{\nu}^{\mu}$ $=\frac{1}{2}\left(\partial u^{\mu} / \partial x^{\nu}-\partial u^{\nu} / \partial x^{\mu}\right), e_{\nu}{ }^{\mu}=\frac{1}{2}\left(\partial u^{\mu} / \partial x^{\nu}+\partial u^{\nu} / \partial x^{\mu}\right)$, $e=\sum e_{v}{ }_{v}$. The derivation of these equations for the statical case is given in references 8 and 9. We have introduced here explicitly the increment of body force $\Delta X^{\nu}$ due to the motion itself this term must be taken into account if we wish to state correctly the equations of motion of a gravitating body.

We consider now various cases of initial stress conditions and examine the corresponding behavior of elastic waves for each case. For simplicity we restrict ourselves to two-dimensional strain.

\section{Uniform hydrostatic pressure}

In this case

$$
S_{11}=S_{22}=\text { const } S_{12}=0 .
$$

Moreover a uniform pressure implies $X=Y=0$. In this case Eqs. (15) become

$$
\begin{aligned}
& \partial s_{11} / \partial x+\partial s_{12} / \partial y=\rho \partial^{2} u / \partial t^{2}, \\
& \partial s_{12} / \partial x+\partial s_{22} / \partial y=\rho \partial^{2} v / \partial t^{2} .
\end{aligned}
$$

These equations are the same as the classical ones for a body in an unstressed state. The influence of the pressure appears only in the elastic coefficients of the material.

\section{Hydrostatic pressure with uniform pressure gradient}

Such a case will occur in a material under the action of gravity when the influence of creep has been acting a sufficiently long time so that the stress condition at every point has become isotropic. This condition must be approximately realized in the earth.

Taking the $y$ axis positive downward we have

$$
X=0, \quad Y=g,
$$

where $g$ is the acceleration of gravity. Also $S_{11}=S_{22}$ and $S_{12}=0$. From (6) we derive

$$
\begin{gathered}
\partial S_{11} / \partial x=\partial S_{22} / \partial y=0, \\
\partial S_{22} / \partial y=\partial S_{11} / \partial y=-\rho g .
\end{gathered}
$$

We assume the specific mass to be uniform ( $\rho=$ constant $)$. With these conditions Eqs. (15) become

$$
\begin{aligned}
& \frac{\partial s_{11}}{\partial x}+\frac{\partial s_{12}}{\partial y}+\rho g \frac{\partial v}{\partial x}=\rho \frac{\partial^{2} u}{\partial t^{2}}, \\
& \frac{\partial s_{12}}{\partial x}+\frac{\partial s_{22}}{\partial y}-\rho g \frac{\partial u}{\partial x}=\rho \frac{\partial^{2} v}{\partial t^{2}}
\end{aligned}
$$

These equations are different from the classical ones for an initially unstressed medium. The additional terms are due to the existence of a pressure gradient. In order to investigate the behavior of the waves for this case, let us assume the stress-strain relations to be Hooke's law for an isotropic medium

$$
\begin{aligned}
& s_{11}=\lambda e+2 G e_{x x}, \\
& s_{22}=\lambda e+2 G e_{y y}, \\
& s_{12}=2 G e_{x y} .
\end{aligned}
$$

By substitution in Eqs. (22) we find

$$
\begin{aligned}
& G \nabla^{2} u+(G+\lambda) \frac{\partial e}{\partial x}+\rho g \frac{\partial v}{\partial x}=\rho \frac{\partial^{2} u}{\partial t^{2}}, \\
& G \nabla^{2} v+(G+\lambda) \frac{\partial e}{\partial y}-\rho g \frac{\partial u}{\partial x}=\rho \frac{\partial^{2} v}{\partial t^{2}}
\end{aligned}
$$


We derive the following equations for the dilatation $e$ and the rotation $\omega$ by the usual method

$$
\begin{gathered}
(2 G+\lambda) \nabla e^{2}+2 \rho g \partial \omega / \partial x=\rho \partial^{2} e / \partial t^{2} \\
G \nabla^{2} \omega-2 \rho g \partial e / \partial x=\rho \partial^{2} \omega / \partial t^{2}
\end{gathered}
$$

These equations show the existence of coupling between longitudinal and transversal waves. This coupling is due to the existence of an initial pressure gradient. The physical meaning of this coupling is as follows. Consider a dilatational plane wave propagating in the horizontal direction. At a point where the material is compressed it is denser and therefore has a negative buoyancy, while in the region of positive dilatation the buoyancy is positive. The dilatational wave is therefore associated with a periodic distribution of vertical buoyancy force which generates a transversal wave. Conversely a transversal wave propagating in the horizontal direction produces a dilatational wave. In this case a portion of the material which is horizontal in the initial state undergoes a rotation and the initial pressure gradient acquires a horizontal component of alternating sign which causes a dilatational wave. The effect increases with the wave-length and we may verify that for the case of the earth it can only acquire an importance in the study of tidal waves with a wave-length of the order of $1000 \mathrm{~km}$ or larger.

\section{Influence of gravity on Rayleigh waves}

It was shown by Rayleigh that elastic waves may propagate along the surface of elastic bodies the amplitude of the wave decreasing exponentially with depth. It is clear that such waves at the surface of the earth must be influenced by gravity. The effect must increase with the wavelength, and also when the rigidity of the medium becomes smaller. In fact, we can imagine materials less and less rigid, such as jelly, in which gravity would have a predominant effect, and consider finally the limiting case of a liquid in which the rigidity is zero and the waves such as ocean waves are due entirely to gravity. As the velocity of the latter depends on the wave-length, we may expect that one of the effects of gravity on Rayleigh waves is to make their velocity of propagation depend on the wave-length. The theory of this effect was first attempted by Bromwich. ${ }^{3}$ It is interesting to verify that his equations may be derived from our general theory provided we introduce the assumption that the initial state of stress is hydrostatic.

We introduce the same simplifying assumption as Bromwich, namely that the material is incompressible. This eliminates the dilatational wave. We then add the assumption that the initial state of stress is a hydrostatic pressure with a pressure gradient due to gravity, and apply therefore the above Eqs. (24). We may write Hooke's law for an incompressible material as follows :

$$
\begin{aligned}
& s_{11}=-p+2 G e_{x x}, \\
& s_{22}=-p+2 G e_{y y}, \\
& s_{12}=2 G e_{x y},
\end{aligned}
$$

where $p$ is the increment of hydrostatic pressure. Substituting these values in Eqs. (24) and taking into account the condition of incompressibility $\partial u / \partial x+\partial v / \partial y=0$ we find

$$
\begin{gathered}
G \nabla^{2} u-\frac{\partial}{\partial x}(p-\rho g v)=\rho \frac{\partial^{2} u}{\partial t^{2}} \\
G \nabla^{2} v-\frac{\partial}{\partial y}(p-\rho g v)=\rho \frac{\partial^{2} v}{\partial t^{2}}
\end{gathered}
$$

Assuming the $x$ axis to coincide with the free surface, the boundary conditions (19) for $y=0$ are $s_{22}=s_{12}=0$ or

$$
\begin{aligned}
& 0=-p+2 G e_{y y}, \\
& 0=2 G e_{x y} .
\end{aligned}
$$

Putting $p_{1}=p-\rho g v$, the wave equation becomes

$$
\begin{gathered}
G \nabla^{2} u-\partial p_{1} / \partial x=\rho \partial^{2} u / \partial t^{2}, \\
G \nabla^{2} v-\partial p_{1} / \partial y=\rho \partial^{2} v / \partial t^{2}
\end{gathered}
$$

and the boundary condition

$$
\begin{aligned}
-p_{1}+2 G e_{y y} & =\rho g v, \\
2 G e_{x y} & =0 .
\end{aligned}
$$

The quantity $p_{1}$ may be interpreted physically as the pressure increment due to the waves at a fixed point $(x, y)$, while $p$ is the pressure at a point originally of coordinates $x, y$ but displaced 
with the material at the coordinates $x+u, y+v$. The case $G=0$ corresponds to a liquid medium and Eqs. (28) become the hydrodynamical equations of an incompressible fluid for small oscillations. Eqs. (28) and (29) are identical with those considered by Bromwich. He also derived Eq. (31) below for the velocity.

We can verify that we may write the following solution for Eqs. (28)

with

$$
\begin{gathered}
u=\left(A l e^{-l y}+C r e^{-r y}\right) \sin (l x-\alpha t), \\
v=\left(A l e^{-l y}+C l e^{-r y}\right) \cos (l x-\alpha t), \\
p_{1}=-A \alpha^{2} \rho e^{-l y} \cos (l x-\alpha t) \\
r=l\left(1-\zeta^{2}\right)^{\frac{1}{2}}, \quad \zeta^{2}=\alpha^{2} \rho / G l^{2} .
\end{gathered}
$$

This solution also verifies the condition $\partial u / \partial x$ $+\partial v / \partial y=0$ of incompressibility. From the boundary conditions (29) we derive

$$
\begin{aligned}
\left(2 G l^{2}-\alpha^{2} \rho+\rho g l\right) A+(2 G l r+\rho g l) C & =0, \\
2 l^{2} A+\left(l^{2}+r^{2}\right) C & =0 .
\end{aligned}
$$

Elimination of $A$ and $C$ yields

$$
\left(2-\zeta^{2}\right)^{2}-\frac{\rho g \zeta^{2}}{G l}=4\left(1-\zeta^{2}\right)^{\frac{1}{2}}
$$

The quantity $\zeta$ in this equation is the ratio of the velocity of the Rayleigh wave $v=\alpha / l$ to the velocity of a shear wave $v_{t}=(G / \rho)^{\frac{1}{2}}$

$$
\zeta=v / v_{t}
$$

Eq. (31) therefore yields the velocity of the Rayleigh wave as a function of the dimensionless parameter $\rho g / G l$. This parameter represents the influence of gravity and it can be seen that the velocity increases with the wave-length $2 \pi / l$, but as shown already by Bromwich the correction for waves at the surface of the earth is small even for very large wave-length.

For a very small wave-length $(l=\infty)$ the equation yields the root $\zeta^{2}=0.912$ which is the value found by Rayleigh. For an increasing wavelength the value of the root increases until $\rho g / G l=1$ for which $\zeta=1$.

Consider, for instance, the case of the earth. We assume $g=981 \mathrm{~cm} / \mathrm{sec}$., $\rho=3 \mathrm{~g} / \mathrm{cm}^{3}, G=(1.5) 10^{11}$ dynes $/ \mathrm{cm}^{2}$ then $\rho g / G l=1$ for a wave-length

$$
2 \pi / l=3200 \mathrm{~km} \text {. }
$$

This shows that even for a wave-length equal to half the earth's radius the increase of velocity is of the order of 5 percent.

The limiting case $\rho g / G l=1$ occurs when the velocity $(g / l)^{\frac{t}{3}}$ of the gravity waves is equal to the velocity $(G / \rho)^{\frac{1}{2}}$ of the shear waves.

It is of interest to investigate what happens when the material becomes less and less rigid and the rigidity $G$ tends to zero. For values $\rho g / G l>1$ the solution above breaks down and it is impossible to satisfy the boundary condition with a real solution. We therefore introduce a solution of the following type:

$$
\begin{gathered}
u=\left(A l e^{-l y}-C \mu \sin \mu y\right) \sin (l x-\alpha t), \\
v=\left(A l e^{-l y}+C l \cos \mu y\right) \cos (l x-\alpha t), \\
p_{1}=-A \alpha^{2} \rho e^{-l y} \cos (l x-\alpha t) \\
\mu=l\left(\frac{\alpha^{2} \rho}{G l}-1\right) .
\end{gathered}
$$

with

Introducing these expressions in the boundary conditions (29) we find

$$
\begin{array}{r}
-2 A l^{2}-C \alpha^{2} \rho / G=0, \\
A\left(\alpha^{2} \rho-2 G l^{2}-\rho g l\right)-C \rho g l=0 .
\end{array}
$$

Elimination of $A$ and $C$ yields

$$
\left(2 G l^{2} / \alpha^{2} \rho-1\right)\left(l g / \alpha^{2}-1\right)=0,
$$

which can be considered as an equation for $l$. There are two solutions. One,

$$
\lg / \alpha^{2}=1,
$$

depends on gravity. The velocity of the corresponding waves is $\alpha / l=(\mathrm{g} / \mathrm{l})^{\frac{1}{2}}$ and is equal to that of the gravity wave. This solution is valid only if $g \rho / G l>1$.

It is a combination of a pure gravity wave with a transversal plane wave coming from the depth of the material and being reflected at the surface at an angle $\tan ^{-1}(l / \mu)$ with the vertical. The relative amplitude of this wave is

$$
\frac{C\left(\mu^{2}+l^{2}\right)^{\frac{1}{2}}}{A l}=-2\left(\frac{G l}{\rho g}\right)^{\frac{1}{2}} .
$$

It decreases with the rigidity. This indicates that a pure gravity wave for wave-lengths or rigidities such that $\rho g / G \lambda>1$ cannot persist, but must lose 
energy by radiation of a transversal wave inside the material. This loss of energy vanishes when $G=0$, i.e., the material becomes a liquid, and we are left with the gravity wave alone.

The other solution of Eq. (33) is

$$
2 G l^{2} / \alpha^{2} \rho=1 .
$$

It is independent of gravity. The reason for this is that $A+C=0$ and the displacement $v=0$ at $y=0$, i.e., the free surface stays a plane. The velocity of this wave is

$$
\alpha / l=(2 G / \rho)^{\frac{1}{2}},
$$

i.e., 40 percent higher than that of a pure transversal wave. Since $A=-C$, such a wave when excited must dissipate very quickly by radiation inside. The phenomena pointed out here will influence those modes of oscillation of the earth which have a wave-length above $1000 \mathrm{~km}$.

\section{Medium under initial vertical compression}

We first consider a state of uniform initial stress and take the principal directions of this initial stress along the $x$ and $y$ axes. Then $S_{12}=0$ and the components $S_{11}, S_{22}$ are constant values of the principal initial stresses. Eqs. (15) become

$$
\begin{aligned}
& \frac{\partial s_{11}}{\partial x}+\frac{\partial s_{12}}{\partial y}+\left(S_{11}-S_{22}\right) \frac{\partial \omega}{\partial y}=\rho \frac{\partial^{2} u}{\partial t^{2}}, \\
& \frac{\partial s_{12}}{\partial x}+\frac{\partial s_{22}}{\partial y}+\left(S_{11}-S_{22}\right) \frac{\partial \omega}{\partial x}=\rho \frac{\partial^{2} v}{\partial t^{2}} .
\end{aligned}
$$

These equations are also different from the classical ones for an unstressed initial state. It is seen that the additional terms depend only on the difference between the principal stresses $S_{11}-S_{22}$ and on the rotation. Hence only the rotational waves are affected by this stress condition.

We assume that the vertical and horizontal directions are directions of elastic symmetry, and consider the general stress-strain relations (17). Because $S_{12}=0$ and the assumption of symmetry we have

$$
B_{13}=B_{31}=B_{23}=B_{32}=0 \text {. }
$$

Hence the stress-strain relations become

$$
\begin{aligned}
& s_{11}=B_{11} e_{x x}+B_{12} e_{y y}, \\
& s_{22}=B_{21} e_{x x}+B_{22} e_{y y}, \\
& s_{12}=B_{33} e_{x y} .
\end{aligned}
$$

From (18) we still have $B_{12}-B_{21}=S_{22}-S_{11}$. Therefore there are four distinct elastic constants in these relations. Introducing these relations in Eqs. (34) we derive

$$
\begin{aligned}
G \nabla^{2} u+\left(B_{12}+G\right) \frac{\partial e}{\partial x}+ & \left(B^{\prime}-2 G\right) \frac{\partial^{2} u}{\partial x^{2}} \\
& +\left(S_{11}-S_{22}\right) \frac{\partial \omega}{\partial y}=\rho \frac{\partial^{2} u}{\partial t^{2}} \\
G \nabla^{2} v+\left(B_{21}+G\right) \frac{\partial e}{\partial y}+ & \left(B^{\prime \prime}-2 G\right) \frac{\partial^{2} v}{\partial y^{2}} \\
& +\left(S_{11}-S_{22}\right) \frac{\partial \omega}{\partial x}=\rho \frac{\partial^{2} v}{\partial t^{2}}
\end{aligned}
$$

with

$$
\begin{gathered}
B_{33}=2 G, \quad B^{\prime}=B_{11}-B_{12}, \quad B^{\prime \prime}=B_{22}-B_{31} \\
e=e_{x x}+e_{y y} .
\end{gathered}
$$

Consider now a transversal plane wave propagation in the horizontal direction

$$
u=0, \quad v=\cos (l x-\alpha t) .
$$

By substitution in Eqs. (36) we find the velocity

$$
V_{h}=\frac{\alpha}{l}=\left(G+\frac{1}{2}\left(S_{11}-S_{22}\right)\right)^{\frac{1}{3}} / \rho^{\frac{1}{3}}
$$

A similar wave propagating in the vertical direction would have the velocity

$$
V_{v}=\left(G-\frac{1}{2}\left(S_{11}-S_{22}\right)\right)^{\frac{1}{2}} / \rho^{\frac{1}{2}} .
$$

For instance if there is a vertical compression $P$ in the material

$$
S_{22}=-P, \quad S_{11}=0,
$$

the velocity in the horizontal direction is greater than in the vertical. This effect is essentially due to the existence of the initial stress, because if this initial stress is zero or if it is a hydrostatic pressure then the two velocities are the same in spite of the elastic anisotropy of the material. 
Note that this phenomenon is the same if we put

$$
S_{22}=0, \quad S_{11}=-P .
$$

The initial condition is then a horizontal tension $-P$ and the velocity of shear waves propagating in the horizontal direction is

$$
V_{h}=\left(\frac{G-\frac{1}{2} P}{\rho}\right)^{\frac{1}{2}} .
$$

If we put $G=0$ in this formula we do not find the velocity in a membrane or a string under tension. This is because the wave in a membrane is not a shear wave but a bending wave. For the viewpoint of the theory of elasticity a bending wave is the combination of two waves of the Rayleigh type at both free boundaries. ${ }^{10}$ If we calculate the velocity of these bending waves and then put $G=0$ we find

$$
V_{h}=(-P / \rho)^{\frac{1}{2}},
$$

which is the velocity of a wave in a membrane under tension $P$.

This analysis shows that propagation and reflections of elastic waves in a material under initial stress must follow laws which cannot be explained by elastic anisotropy or a change in elastic constants. In fact, because the velocity of propagation depends on the total initial shear, a discontinuity in shear may produce a reflection even if there is no discontinuity in elastic constants.

10 S. P. Timoshenko, "On the transverse vibrations of bars of uniform cross section." Phil. Mag. 43, 125 (1922).

\title{
Equations of Finite Differences Applied to Torsional Oscillations of Crankshafts*
}

\author{
Maurice A. Biot \\ Columbia University, New York, New York
}

(Received April 8, 1940)

From the viewpoint of torsional oscillations an internal combustion engine with a long crankshaft is generally considered to be equivalent to a uniform shaft carrying equidistant identical disks. It is here shown that advantage can be taken of the regularity of such a system to simplify the calculation of torsional oscillations. This is done by applying a mathematical method known as the calculus of finite differences. The procedure leads to a frequency equation (2.7) of remarkable symmetry in which appear as parameter the number $n$ of cylinders in line and two simple functions $K_{1}$ and $K_{2}$ of the frequency which characterize the dynamical properties of the machines coupled at both ends of the crankshaft. These characteristic functions are

\section{Mechanical Impedance and Dynamic Modulus}

$\coprod \mathrm{N}$ $\checkmark$ the theory of electric networks the concept of impedance has proved to be a highly useful tool for both the analytical treatment and the comprehension of electrical phenomena. Its use has been extended to the field of mechanics by the introduction of so-called equivalent networks or, as in acoustics, by defining the mechanical impedance as the ratio of force to velocity. The

\footnotetext{
* Publication assisted by the Ernest Kempton Adams Fund for Physical Research of Columbia University.
}

of the nature of mechanical impedances, but due to their physical interpretation as a spring modulus. (or spring constant) generalized to dynamic phenomena, the appellation dynamic modulus is being preferably used in the present paper. The concept of dynamic modulus is briefly introduced in the first section, while the second deals with the establishment of the frequency equation and an artifice for its rapid graphical solution avoiding the necessity of plotting an oscillatory function. Numerical applications to Diesel engines are treated in the last section. An example is also given of an extreme case where the fundamental frequency has a very low value and a special method is used for the calculation of this frequency.

latter definition is very useful in compound electromechanical systems and in those for which the amount of dissipated or radiated energy is one of the important features. However, in systems without or with negligible dissipation constituted, for instance, by a combination of masses and springs, it seems preferable to introduce as mechanical impedance the ratio of force to displacement. When there is no dissipation this ratio is a real quantity which can be either positive or negative. It generalizes the concept of spring constant to the case of harmonic mo- 